TRANSACTIONS OF THE

AMERICAN MATHEMATICAL SOCIETY

Volume 353, Number 11, Pages 4545-4565

S 0002-9947(01)02834-3

Article electronically published on May 9, 2001

\title{
BOUNDEDNESS AND DIFFERENTIABILITY FOR NONLINEAR ELLIPTIC SYSTEMS
}

\author{
JANA BJÖRN
}

\begin{abstract}
We consider the elliptic system $\operatorname{div}\left(\mathcal{A}^{j}(x, u, \nabla u)\right)=\mathcal{B}^{j}(x, u, \nabla u)$, $j=1, \ldots, N$, and an obstacle problem for a similar system of variational inequalities. The functions $\mathcal{A}^{j}$ and $\mathcal{B}^{j}$ satisfy certain ellipticity and boundedness conditions with a $p$-admissible weight $w$ and exponent $1<p \leq 2$. The growth of $\mathcal{B}^{j}$ in $|\nabla u|$ and $|u|$ is of order $p-1$. We show that weak solutions of the above systems are locally bounded and differentiable almost everywhere in the classical sense.
\end{abstract}

\section{INTRODUCTION}

In this paper we study weak solutions $u=\left(u^{1}, \ldots, u^{N}\right)$ of the following system of second-order quasilinear elliptic equations in divergence form

$$
\operatorname{div}\left(\mathcal{A}^{j}(x, u, \nabla u)\right)=\mathcal{B}^{j}(x, u, \nabla u), \quad j=1, \ldots, N,
$$

in a bounded domain $\Omega \subset \mathbf{R}^{n}, n \geq 2$.

We also consider the obstacle problem for the following system of variational inequalities:

$$
\sum_{j=1}^{N} \int_{\Omega} \mathcal{A}^{j}(x, u, \nabla u) \cdot \nabla\left(u^{j}-v^{j}\right) d x+\sum_{j=1}^{N} \int_{\Omega} \mathcal{B}^{j}(x, u, \nabla u)\left(u^{j}-v^{j}\right) d x \leq 0
$$

for all $v=\left(v^{1}, \ldots, v^{N}\right)$ satisfying $v \geq \psi$ in $\Omega$ and $v=u$ on $\partial \Omega$. The solution $u=\left(u^{1}, \ldots, u^{N}\right)$ is required to satisfy $u \geq \psi$ in $\Omega$.

In both cases, the functions $\mathcal{A}^{j}$ and $\mathcal{B}^{j}$ satisfy certain ellipticity and boundedness conditions with a $p$-admissible weight $w$ and exponent $1<p \leq 2$. The growth of $\mathcal{B}^{j}$ in $|\nabla u|$ and $|u|$ is of order $p-1$. The exact assumptions on the functions $\mathcal{A}^{j}$ and $\mathcal{B}^{j}$ and some examples are given in Section 3

We shall show that weak solutions of the system (1.1) as well as solutions of the obstacle problem (1.2) are locally bounded in the domain $\Omega$, see Theorem 3.2 For applications it would be more natural to allow the right-hand sides $\mathcal{B}^{j}$ to grow as $|\nabla u|^{p}$ rather then $|\nabla u|^{p-1}$. However, already for one equation, the assumption

Received by the editors December 8, 1999 and, in revised form, November 20, 2000.

2000 Mathematics Subject Classification. Primary 35J70; Secondary 35B35, 35B65, 35D10, $35 \mathrm{~J} 60,35 \mathrm{~J} 85$

Key words and phrases. Elliptic system, p-admissible weight, obstacle problem, local boundedness, differentiability a.e.

The results of this paper were obtained while the author was visiting the University of Michigan, Ann Arbor, on leave from the Linköping University. The research was supported by grants from the Swedish Natural Science Research Council, the Knut and Alice Wallenberg Foundation and Gustaf Sigurd Magnusons fond of the Royal Swedish Academy of Sciences.

(C)2001 American Mathematical Society 
$|\mathcal{B}| \leq C|\nabla u|^{p}$ does not guarantee boundedness of solutions, see e.g. pp. $25-27$ in Ladyzhenskaya-Ural'tseva [24].

For $N=1$ and $w=1$, it is well known that weak solutions of a single equation with $1<p<\infty$ are locally bounded (and locally Hölder continuous), see e.g. the fundamental paper by Serrin 33. or Chapter 4.7 in Ladyzhenskaya-Ural'tseva [24. Boundedness of solutions of the obstacle problem for $N=1$ and $w=1$ has been proved under various assumptions on $\mathcal{A}, \mathcal{B}$ and $\psi$ in e.g. Lewy-Stampacchia [26], Frehse-Mosco [8], Ural'tseva [38, Michael-Ziemer [28] and by the author in [21]. Properties of solutions of weighted quasilinear equations with $1<p<\infty$ and the corresponding obstacle problem have been extensively studied in the monograph by Heinonen-Kilpeläinen-Martio [18]. Let us also mention a paper by Naselli Ricceri [30, in which $L^{\infty}$-bounds for solutions of an $A_{2}$-degenerate variational inequality have been obtained.

On the other hand, for elliptic systems with $N>1$, examples of unbounded weak solutions have been given by e.g. De Giorgi [5] and Frehse [7, thus there is no hope for extending the above-mentioned results about local boundedness to systems in general. There even exist systems whose solutions are unbounded in every neighbourhood of every point, see Remark on p. 41 in John-Malý-Stará [22].

Local boundedness has been proved for systems whose principal part consists of a uniformly elliptic operator times the identity matrix, see e.g. Chapter 7.2 in Ladyzhenskaya-Ural'tseva [24] for $p=2$ and Landes [25] for $p \geq 2$. NečasStará [31] considered a nondiagonal uniformly elliptic system, which turns into a diagonal one at the points where the solution is sufficiently large. For $p=2$, it is known that solutions of linear systems with continuous coefficients are locally Hölder continuous, see e.g. Chapter III.3 in Giaquinta [11]. For general systems, most results are in terms of partial regularity, i.e. except for a closed set with zero measure, see e.g. Morrey [29], Giusti-Miranda [14], Giusti [13], GiaquintaModica [12] and Giaquinta [11] for systems of equations and Fuchs [10] for some systems of variational inequalities.

In Meier [27], boundedness of weak solutions of very general nondiagonal systems was proved under the assumption that the so-called indicator function

$$
\sum_{i, j=1}^{N} \frac{u^{i} u^{j}}{|u|^{2}} \mathcal{A}^{j}(x, u, \nabla u) \cdot \nabla u^{i}
$$

is nonnegative at the points where $|u|$ becomes large. Even though this condition is always satisfied for a certain class of systems (containing e.g. certain diagonal systems, harmonic maps between Riemannian manifolds and other special structures), it is difficult to verify this condition in general.

In this paper we are concerned with systems which, roughly speaking, are not too far from being diagonal. This is expressed essentially by requiring that

$$
\mathcal{A}^{j}(x, u, q) \cdot q^{j} \geq\left(\left|q^{j}\right|^{p}-d(x)|u|^{p}-f(x)\right) w(x), \quad j=1, \ldots, N,
$$

which is stronger than the ellipticity condition $\sum_{j=1}^{N} \mathcal{A}^{j} \cdot q^{j} \geq\left(|q|^{p}-d|u|^{p}-f\right) w$, considered with $w=1$ in Meier [27. On the other hand, we do not require any assumptions involving properties of a particular solution, such as the indicator function. Moreover, we allow the operators to be degenerate with a $p$-admissible weight $w$, thus extending even the case $N=1$ both for equations and variational inequalities (and all $p>1$, see Remark 5.1). 
Our method is based on De Giorgi type estimates of $|\nabla U|^{p}$, where

$$
U=\max \left\{\left|u^{1}\right|, \ldots,\left|u^{N}\right|\right\},
$$

and makes use of the test functions $\varphi^{j}=(\max (U-k, 0) / U)^{p}\left(u^{j}-U\right) \eta^{p}$, where $\eta$ is a cut-off function. It applies both to systems of equations and systems of variational inequalities. In fact, the system (1.1) can be considered as a special case of (1.2) with $\psi^{j}=-\infty, j=1, \ldots, N$, and by letting only some $\psi^{j}=-\infty$, we can consider systems consisting of both equations and inequalities.

In the last section, following a method of Reshetnyak-Hajłasz-Strzelecki based on the difference quotients $u_{h}(X)=\left(u\left(x_{0}+h X\right)-u\left(x_{0}\right)\right) / h$, our boundedness estimates are used to prove that weak solutions of the system (1.1) and the obstacle problem (1.2) are differentiable almost everywhere in the classical sense, see Theorem 6.7. For this, we do not impose any smoothness assumptions on the functions $\mathcal{A}^{j}$ and $\mathcal{B}^{j}$, the essential ingredient are good $L^{\infty}$-estimates for solutions (depending only on the system and on the $L^{p}$-norm of the solutions).

Bojarski 4] showed that weak solutions of a single unweighted linear elliptic equation are differentiable a.e. in $\Omega$. This gives an additional geometrical information about the solutions in addition to their Hölder continuity. For unweighted quasilinear elliptic equations of the type studied here $(N=1$ and $w=1)$, differentiability a.e. of weak solutions was proved by Reshetnyak in [32. Reshetnyak's proof is based on the Hölder continuity of the solutions. Hajłasz-Strzelecki [17] showed that using Bojarski's method one can simplify Reshetnyak's proof so that it uses local boundedness rather than the Hölder continuity of weak solutions. In fact Karch-Ricciardi [23] showed by essentially the same method that differentiability a.e. can be obtained even for equations whose weak solutions are not Hölder continuous.

The author showed in Ježková [21] that the method of Reshetnyak-HajłaszStrzelecki can be extended to unweighted variational inequalities $(N=1$ and $w=1)$ and also to bounded solutions of equations and inequalities, whose right-hand side grows quadratically (for $p=2$ ) in $|\nabla u|$. At this point we would like to mention that the assumption (2.2) in [21] about smallness of the coefficient $c(x)$ is superfluous it can be replaced by $\sup _{B\left(x_{0}, r\right)} c<1 / \operatorname{osc}_{B\left(x_{0}, r\right)} u$ for some $r>0$ and as bounded solutions of a single equation with quadratic growth in $|\nabla u|$ are locally Hölder continuous, this condition is always satisfied.

Let us briefly discuss some cases in which the method of Reshetnyak-HajłaszStrzelecki can be applied and those where it fails. The partial regularity $u \in$ $C^{0, \alpha}(\Omega \backslash \Sigma)$ with $|\Sigma|=0$, which has been proved for large classes of systems, is of qualitative character and does not give good enough estimates of $\|u\|_{L^{\infty}}$ for the method to be applied. Also, the $L^{\infty}$-estimates obtained by Landes [25] for solutions of nonlinear elliptic diagonal systems with $p \geq 2$ depend on ess $\sup _{\partial \Omega}\left|u^{j}\right|$ and cannot therefore be used to prove differentiability a.e. of the solutions. Since the positivity of the indicator function (1.3) for $u$ does not in general imply the positivity of the indicator function for the difference quotients $u_{h}$, the general boundedness results for $1<p \leq n$ in Meier [27] cannot be used to obtain differentiability a.e. of solutions. On the other hand, some special structures considered in Corollary 1 in 27] can be treated by the Reshetnyak-Hajłasz-Strzelecki method yielding differentiability a.e. In particular this applies to diagonal systems with $p=2$ and the right-hand side growing linearly in the gradient (which are covered by our assumptions) or satisfying $|\mathcal{B}| \leq c|\nabla u|^{2}+f$, where $f \in L^{n /(2-\varepsilon)}$ and $c<1 / 2 \sup _{\Omega}|u|$. 
Let us also mention some results concerning higher regularity, which have been proved under various smoothness assumptions on the functions $\mathcal{A}^{j}$ and $\mathcal{B}^{j}$. Solutions of linear systems with Hölder continuous coefficients $(p=2)$ have Hölder continuous first derivatives, see e.g. Chapter III.3 in Giaquinta [11]. Partial $C^{1, \alpha}$ regularity for bounded solutions of the elliptic system (1.1) with $p=2, \mathcal{A}^{j}$ Hölder continuous in $x$ and $u$ and continuously differentiable with respect to $q,|\mathcal{B}| \leq$ $c|\nabla u|^{2}+b$ and $c<1 / 2 \sup _{\Omega}|u|$ has been proved by Giaquinta-Modica [12].

For $p \geq 2$, Uhlenbeck [37] showed that solutions of the system $\operatorname{div}\left(a\left(|\nabla u|^{2}\right) \nabla u^{j}\right)$ $=0$, with a positive function $a$, which grows as $t^{p / 2-1}$ and satisfies $a(t)+2 a^{\prime}(t) t>$ 0 , are of class $C^{1, \alpha}$. For $p=2$, Ivert [20] proved $C^{1, \alpha}$-regularity for bounded solutions of the system $\operatorname{div}\left(A\left(x, u,|\nabla u|^{2}\right) \nabla u^{j}\right)=\mathcal{B}^{j}$ with a smooth function $A$ and $\mathcal{B}^{j}$ growing quadratically in the gradient (with limited speed). For $p>1$, Tolksdorf 36] further generalizes these results to quasilinear systems whose lefthand side is the $\nabla u$-directional derivative of the functional

$$
u \longmapsto \int_{\Omega} F(x, \rho(\nabla u)) d x,
$$

where $F(x, t)$ grows as $t^{p / 2}$ and

$$
\rho(q)=\sum_{\alpha, \beta=1}^{n} \sum_{i, j=1}^{N} \gamma_{\alpha \beta}(x) g^{i j}(x) q_{\alpha}^{i} q_{\beta}^{j}
$$

is a quadratic form with $C^{1}$-coefficients satisfying $\rho(q) \geq \gamma_{0}|q|^{2}$ for all $x$ and $q$.

Let us briefly compare our assumptions with those in Tolksdorf [36]. The ellipticity condition in [36] is essentially $\sum_{j=1}^{N} \mathcal{A}^{j}(x, q) \cdot q^{j} \geq \lambda(\kappa+|q|)^{p-2}|q|^{2}$, which is clearly weaker then (1.4). At the same time the functions $A, g_{i j}$ and $\gamma^{\alpha \beta}$ in [36] are required to be at least differentiable with certain bounds on the derivatives. Hence, the results of 36 apply e.g. to the minima of the functional (1.5) with smooth coefficients, in particular to the vector-valued $p$-Laplacian and its smooth perturbations. Such systems do not satisfy (1.4). On the other hand, the special form of the functions $\mathcal{A}^{j}$ in 36 disqualifies systems in which the left-hand sides differ considerably from one equation to another, such as those given in Examples 3.4 and 3.5 Also, we allow $\mathcal{A}^{j}$ and $B^{j}$ which are not differentiable in any variable, such as those in Example 3.6

As for systems of variational inequalities, Hildebrandt-Widman [19] consider the case $p=2$ with $\mathcal{A}^{j}(x, u, \nabla u)=A(x, u) \nabla u^{j}+g^{j}(x),|\mathcal{B}| \leq c|\nabla u|^{2}+f$ and the constraint $u: \Omega \rightarrow M \subset \mathbf{R}^{N}$. Under certain smoothness assumptions on the coefficients, they show that if $c \operatorname{diam} M<1$, then $u \in C^{1, \alpha}(\Omega)$. In [9], Fuchs proves partial $C^{1, \alpha}$-regularity for minimizers of integral functionals with $C^{1}$-coefficients, growth of order $p \geq 2$ and the constraint $u: \Omega \rightarrow M \subset \mathbf{R}^{N}$. For $1<p<2$, partial $C^{1, \alpha}$-regularity for minimizers of integral functionals depending on $|\nabla u|$ has been proved by Acerbi-Fusco [1].

\section{Weighted Sobolev spaces}

In this section we remind the reader of the definition of $p$-admissible weights and weighted Sobolev spaces. Let $B\left(x_{0}, r\right)$ denote the open ball in $\mathbf{R}^{n}$ with the center $x_{0}$ and radius $r$. By $C_{0}^{\infty}(\Omega)$ we mean the space of infinitely many times differentiable functions on $\Omega$ with compact support in $\Omega$. By $|E|$ we denote the Lebesgue measure of the set $E$. Unless otherwise stated, the letter $C$ will denote a 
positive constant whose exact value is unimportant and may change even within a line. Where necessary, we shall specify the dependence of $C$ on various parameters.

Let $1<p<\infty$ and let $w$ be a nonnegative locally Lebesgue integrable function in $\mathbf{R}^{n}$ and let $\mu$ denote the Radon measure defined by $\mu(E)=\int_{E} w(x) d x$, i.e. $d \mu=w d x$. We shall assume that $w$ is $p$-admissible, i.e. that the following conditions are satisfied (see Theorem 2 in Hajłasz-Koskela [15]).

(i) Doubling condition: $0<w<\infty$ a.e. in $\mathbf{R}^{n}$ and there is a constant $c_{1}>0$ such that

$$
\mu\left(B\left(x_{0}, 2 r\right)\right) \leq c_{1} \mu\left(B\left(x_{0}, r\right)\right)
$$

for all balls $B\left(x_{0}, r\right) \subset \mathbf{R}^{n}$.

(ii) Weighted Poincaré inequality: There is a constant $c_{2}>0$ such that for all balls $B=B\left(x_{0}, r\right) \subset \mathbf{R}^{n}$ and $\varphi \in C^{\infty}\left(B\left(x_{0}, 2 r\right)\right)$,

$$
f_{B}\left|\varphi-\varphi_{B}\right| d \mu \leq c_{2} r\left(f_{B\left(x_{0}, 2 r\right)}|\nabla \varphi|^{p} d \mu\right)^{1 / p}
$$

where $\varphi_{B}=f_{B} \varphi d \mu$. Here, and in what follows, the symbol $f$ denotes the meanvalue integral

$$
f_{B} \varphi d \mu=\frac{1}{\mu(B)} \int_{B} \varphi d \mu .
$$

It is well known that Muckenhoupt $A_{p}$ weights are $p$-admissible. Other examples of $p$-admissible weights can be found in e.g. [3].

It is a recent result of Hajłasz-Koskela (see Theorem 2 in 15 or Theorem 13.1 in [16]) that the above two conditions imply the weighted Sobolev inequality

$$
\left(f_{B}|\varphi|^{p \kappa} d \mu\right)^{1 / p \kappa} \leq c_{3} r\left(f_{B}|\nabla \varphi|^{p} d \mu\right)^{1 / p}
$$

for some $\kappa>1$ and $c_{3}>0$ (depending only on $p, c_{1}$ and $c_{2}$ ), all balls $B=$ $B\left(x_{0}, r\right) \subset \mathbf{R}^{n}$ and all $\varphi \in C_{0}^{\infty}(B)$. Earlier, in e.g. Section 1.1 in HeinonenKilpeläinen-Martio [18], the Sobolev inequality together with another condition (which also turned out to be a consequence of doubling and the Poincaré inequality) was required in the definition of $p$-admissible weights.

By $L^{p}(\Omega, \mu)$ we denote the space of functions on $\Omega$ whose $p$-th power is integrable with respect to the measure $\mu$. If $\mu$ is the Lebesgue measure, we write just $L^{p}(\Omega)$. The weighted Sobolev space $H_{0}^{1, p}(\Omega, \mu)$ is the closure of $C_{0}^{\infty}(\Omega)$ with respect to the norm

$$
\|\varphi\|_{H^{1, p}(\Omega, \mu)}=\left(\int_{\Omega}|\varphi|^{p} d \mu\right)^{1 / p}+\left(\int_{\Omega}|\nabla \varphi|^{p} d \mu\right)^{1 / p}
$$

and $H^{1, p}(\Omega, \mu)$ is the closure of $\left\{\varphi \in C^{\infty}(\Omega):\|\varphi\|_{H^{1, p}(\Omega, \mu)}<\infty\right\}$ in the $H^{1, p}(\Omega, \mu)$ norm. In other words, $u \in H^{1, p}(\Omega, \mu)\left(u \in H_{0}^{1, p}(\Omega, \mu)\right)$ if and only if $u \in L^{p}(\Omega, \mu)$ and there exist $\varphi_{j} \in C^{\infty}(\Omega)\left(\varphi_{j} \in C_{0}^{\infty}(\Omega)\right)$ and a vector-valued function $\nabla u$ such that $\varphi_{j} \rightarrow u$ and $\nabla \varphi_{j} \rightarrow \nabla u$ in $L^{p}(\Omega, \mu)$, as $j \rightarrow \infty$. If $w^{1 /(1-p)}$ is locally integrable then $\nabla u$ is the distributional gradient of $u$.

Note that the weighted Sobolev and Poincaré inequalities hold for functions in $H_{0}^{1, p}(B, \mu)$ and $H^{1, p}(B, \mu)$, respectively. We shall also need the fact that the 
weighted Sobolev spaces are lattices, i.e. if $\varphi$ and $\eta$ belong to $H^{1, p}(\Omega, \mu)$, then so do $|\varphi|, \max \{\varphi, \eta\}$ and $\min \{\varphi, \eta\}$ and

$$
\nabla \max \{\varphi, \eta\}(x)= \begin{cases}\nabla \varphi(x) & \text { if } \varphi(x) \geq \eta(x), \\ \nabla \eta(x) & \text { if } \eta(x)>\varphi(x),\end{cases}
$$

similarly for $\nabla \min \{\varphi, \eta\}$. Moreover if $\varphi_{i} \rightarrow \varphi$ and $\eta_{i} \rightarrow \eta$ in $H^{1, p}(\Omega, \mu)$, then

$$
\begin{aligned}
\max \left\{\varphi_{i}, \eta_{i}\right\} & \rightarrow \max \{\varphi, \eta\} \quad \text { and } \\
\min \left\{\varphi_{i}, \eta_{i}\right\} & \rightarrow \min \{\varphi, \eta\} \quad \text { in } H^{1, p}(\Omega, \mu) .
\end{aligned}
$$

For more about weighted Sobolev spaces we refer the reader to Chapter 1 in Heinonen-Kilpeläinen-Martio [18].

\section{Formulation of the PROBlem AND the MAIN RESUlt}

We are now ready to state the exact assumptions about the systems (1.1) and (1.2). In what follows, $u=\left(u^{1}, \ldots, u^{N}\right)$ will be a vector-valued function on $\Omega$. By

$$
\nabla u=\left(\nabla u^{j}\right)_{j=1, \ldots, N}=\left(\partial_{\alpha} u^{j}\right)_{j=1, \ldots, N, \alpha=1, \ldots, n},
$$

we denote the gradient of $u$. By $u \in X^{N}$ we mean that the scalar components of $u$ belong to the function space $X$ and $\|u\|_{X^{N}}$ stands for $\left\|u^{1}\right\|_{X}+\cdots+\left\|u^{N}\right\|_{X}$.

We assume that $\mathcal{A}^{j}: \Omega \times \mathbf{R}^{N} \times \mathbf{R}^{n N} \rightarrow \mathbf{R}^{n}$ and $\mathcal{B}^{j}: \Omega \times \mathbf{R}^{N} \times \mathbf{R}^{n N} \rightarrow \mathbf{R}$ are functions measurable in the first variable and continuous in the other variables. We shall require that $\mathcal{A}^{j}$ and $\mathcal{B}^{j}, j=1, \ldots, N$, satisfy the following boundedness and ellipticity conditions with a $p$-admissible weight $w$,

$$
\begin{aligned}
\left|\mathcal{A}^{j}(x, u, q)\right| & \leq\left(a|q|^{p-1}+b(x)|u|^{p-1}+e(x)\right) w(x), \\
\left|\mathcal{B}^{j}(x, u, q)\right| & \leq\left(c(x)|q|^{p-1}+d(x)|u|^{p-1}+f(x)\right) w(x), \\
\mathcal{A}^{j}(x, u, q) \cdot q^{j} & \geq\left(\left|q^{j}\right|^{p}-d(x)|u|^{p}-f(x)\right) w(x)
\end{aligned}
$$

for a.e. $x \in \Omega$, all $u \in \mathbf{R}^{N}$ and all $q=\left(q^{j}\right)_{j=1, \ldots, N}=\left(q_{\alpha}^{j}\right)_{j=1, \ldots, N, \alpha=1, \ldots, n} \in \mathbf{R}^{n N}$. Here $|\cdot|$ stands for the Euclidean norm in the respective space, $a \geq 1$ is a constant and $b, c, d, e$ and $f$ are nonnegative functions such that $b^{p /(p-1)}, c^{p}, d, e^{p /(p-1)}$ and $f$ belong to $L^{\tau}(\Omega, \mu)$, where $\tau=\kappa /(\kappa-1-\varepsilon), 0<\varepsilon \leq 1-1 / \kappa$, and $\kappa$ is the exponent from the weighted Sobolev inequality.

Remark 3.1. In the unweighted case $w=1$, we have $\tau=n /\left(p-\varepsilon^{\prime}\right)$, where $\varepsilon^{\prime}=$ $\varepsilon(n-p)$. Thus, the structure conditions (3.1) correspond to those considered by Serrin 33 for $N=1$, except that we require $b, e \in L^{n /(p-1)+\varepsilon^{\prime \prime}}$ rather than $b, e \in$ $L^{n /(p-1)}$.

The generality of the functions $\mathcal{A}^{j}$ and $\mathcal{B}^{j}$ requires that the system (1.1) be understood in the weak sense, i.e. through the integral identity

$$
\sum_{j=1}^{N} \int_{\Omega} \mathcal{A}^{j}(x, u, \nabla u) \cdot \nabla \varphi^{j} d x+\sum_{j=1}^{N} \int_{\Omega} \mathcal{B}^{j}(x, u, \nabla u) \varphi^{j} d x=0,
$$

which should hold for all $\varphi=\left(\varphi^{1}, \ldots, \varphi^{N}\right) \in C_{0}^{\infty}(\Omega)^{N}$. The solution $u$ is considered in the weighted Sobolev space $H^{1, p}(\Omega, \mu)^{N}$. It follows from the weighted Sobolev inequality that $v \in L_{\mathrm{loc}}^{p \kappa}(\Omega, \mu)^{N}$ for all $v \in H^{1, p}(\Omega, \mu)^{N}$ and the structure conditions (3.1) together with a standard approximation argument then imply that (3.2) holds for all $\varphi \in H_{0}^{1, p}(\Omega, \mu)^{N}$ with compact support in $\Omega$. 
As for the obstacle problem (1.2), let $u_{0}=\left(u_{0}^{1}, \ldots, u_{0}^{N}\right) \in H^{1, p}(\Omega, \mu)^{N} \cap$ $L^{p \kappa}(\Omega, \mu)^{N}$ and $\psi=\left(\psi^{1}, \ldots, \psi^{N}\right)$ be fixed vector-valued functions and define a convex subset of $H^{1, p}(\Omega, \mu)^{N}$ by

$$
K=\left\{u \in H^{1, p}(\Omega, \mu)^{N}: u-u_{0} \in H_{0}^{1, p}(\Omega, \mu)^{N} \text { and } u \geq \psi \text { a.e. in } \Omega\right\} .
$$

Then $u=\left(u^{1}, \ldots, u^{N}\right) \in K$ is a solution of the obstacle problem (1.2) if

$$
\sum_{j=1}^{N} \int_{\Omega} \mathcal{A}^{j}(x, u, \nabla u) \cdot \nabla\left(u^{j}-v^{j}\right) d x+\sum_{j=1}^{N} \int_{\Omega} \mathcal{B}^{j}(x, u, \nabla u)\left(u^{j}-v^{j}\right) d x \leq 0
$$

holds for all $v=\left(v^{1}, \ldots, v^{N}\right) \in K$. As $u_{0} \in L^{p \kappa}(\Omega, \mu)^{N}, u-u_{0} \in H_{0}^{1, p}(\Omega, \mu)^{N}$ and $u-v \in H_{0}^{1, p}(\Omega, \mu)^{N}$, the weighted Sobolev inequality applied to some ball containing $\Omega$ implies that the functions $u$ and $u-v$ belong to $L^{p \kappa}(\Omega, \mu)^{N}$. This together with the structure conditions (3.1) guarantees that all the integrals in (3.3) are finite.

Our main result is contained in the following theorem.

Theorem 3.2. Let $B\left(x_{0}, R\right) \subset \Omega, R \leq 1$, and suppose that the structure conditions (3.1) are satisfied with a $p$-admissible weight $w, 1<p \leq 2$. Consider one of the following two cases:

(a) $u \in H^{1, p}(\Omega, \mu)^{N}$ is a weak solution of the system (3.2), or

(b) $u \in K$ is a solution of the obstacle problem (3.3) and $\operatorname{ess}_{\sup } \operatorname{su}_{B\left(x_{0}, R\right)} \psi^{j}<\infty$ for $j=1, \ldots, N$.

Then

$$
\operatorname{ess}_{\sup _{B\left(x_{0}, R / 2\right)}}|u| \leq 2(\Psi+m)+C\left(f_{B\left(x_{0}, R\right)}|u|^{p} d \mu\right)^{1 / p},
$$

where

$$
\begin{aligned}
\Psi & =\max \left\{0, \operatorname{ess} \sup _{B\left(x_{0}, R\right)} \psi^{j} ; j=1, \ldots, N\right\} \\
m & =\left\|e^{p /(p-1)}+f\right\|_{L^{\tau}\left(B\left(x_{0}, R\right)\right)}^{1 / p}+\|f\|_{L^{\tau}\left(B\left(x_{0}, R\right)\right)}^{1 /(p-1)} \\
C & =C^{\prime}\left(1+\left(f_{B\left(x_{0}, R\right)}\left(b^{p /(p-1)}+c^{p}+d\right)^{\tau} d \mu\right)^{1 / \tau}\right)^{\kappa / p \varepsilon}
\end{aligned}
$$

$\tau=\kappa /(\kappa-1-\varepsilon)$, and the constant $C^{\prime}$ depends only on $p, N, a, \varepsilon$, the doubling constant $c_{1}$ and the constants $c_{3}$ and $\kappa$ from the weighted Sobolev inequality.

As a consequence of Theorem 3.2 we show in the last section that if the obstacle $\psi$ is differentiable a.e., then a representative of the weak solution $u$ is differentiable a.e. in $\Omega$ in the classical sense, see Theorem 6.7.

Example 3.3. Perhaps the simplest example of system (1.1) which does not fall apart into separate equations is

$$
\Delta_{p} u^{j}:=\operatorname{div}\left(\left|\nabla u^{j}\right|^{p-2} \nabla u^{j}\right)=|u|^{p-2} u^{j},
$$

which is the Euler-Lagrange system of the functional

$$
u \longmapsto \int_{\Omega}\left(\sum_{j=1}^{N}\left|\nabla u^{j}\right|^{p}+|u|^{p}\right) d x
$$

which is equivalent to the $H^{1, p}$-norm of $u$. Of course, the right-hand side $\mathcal{B}^{j}=$ $|u|^{p-2} u^{j}$ of each equation in (3.4) satisfies $\mathcal{B}^{j} \operatorname{sign} u^{j} \geq 0$ and Theorem 4.7.1 in 
Ladyzhenskaya-Ural'tseva 24] applied to each equation separately yields estimates for $\operatorname{ess}_{\sup _{\Omega}}\left|u^{j}\right|$ in terms of $\left\|u^{j}\right\|_{L^{p}(\Omega)}$, ess $\sup _{\partial \Omega}\left|u^{j}\right|, p, n$ and $|\Omega|$. Note, however, that due to the dependence on $\operatorname{ess}_{\sup }\left|u^{j}\right|$, this estimate cannot be used to prove the differentiability a.e. of $u$, as in the last section.

More generally, if $w=1, \mathcal{A}^{j}=\mathcal{A}^{j}\left(x, u, \nabla u^{j}\right)$ in (1.1) does not depend on $\nabla u^{i}$, $i \neq j$, the coefficients $b, c, d$ in (3.1) are constant (or $L^{\infty}$ ), $n \leq 4$ and $\frac{1}{2} n<p \leq 2$ (or $1<p \leq 2$ if $b=d=0$ ), then $L^{\infty}$-estimates for $u^{j}$ (in terms of $\left\|u^{j}\right\|_{L^{p}}$ and the parameters of the system) follow from Serrin's theorem for equations (Theorem 1 in [33]). Indeed, in this case we have $\left|\mathcal{B}^{j}\right| \leq f_{1}$, where $f_{1}=c|\nabla u|^{p-1}+d|u|^{p-1}+f$ belongs to $L^{n /(p-\varepsilon)}$, whenever $p^{2}>n(p-1)$, i.e. for e.g. $n \leq 4$ and $1<p \leq 2$. Similarly, $\left|\mathcal{A}^{j}\right| \leq a\left|q^{j}\right|^{p}+e_{1}$ and $\mathcal{A}^{j} \cdot q^{j} \geq\left|q^{j}\right|^{p}-f_{2}$, where $e_{1}=b|u|^{p-1}+e$ and $f_{2}=d|u|^{p}+f$. By the Sobolev inequality, $e_{1}^{p /(p-1)}$ and $f_{2}$ belong to $L^{n /(p-\varepsilon)}$, whenever $n /(n-p)>n / p$, i.e. if $p>\frac{1}{2} n$.

Note, however, that under the assumption $b, c, d \in L^{\infty}$, our results allow us to treat systems (1.1) and (1.2) with higher growth, such as

$$
\begin{aligned}
\left|\mathcal{A}^{j}(x, u, q)\right| & \leq\left(a|q|^{p-1}+b|u|^{\beta-1}+e(x)\right) w(x), \\
\left|\mathcal{B}^{j}(x, u, q)\right| & \leq\left(c|q|^{\gamma-1}+d|u|^{\delta-1}+f(x)\right) w(x), \\
\mathcal{A}^{j}(x, u, q) \cdot q^{j} & \geq\left(\left|q^{j}\right|^{p}-d|u|^{\delta}-f(x)\right) w(x)
\end{aligned}
$$

and $\beta<p(n-1) /(n-p), \gamma<p+p / n, \delta<p n /(n-p)$.

Next, we give some examples of systems which cannot be treated by the scalar theory as in Example 3.3, but which are covered by our assumptions. The structural conditions (3.1) can be regarded as perturbations of diagonal systems. Clearly, there is no need to restricting ourselves to Euler-Lagrange equations of integral functionals. In particular, the right-hand sides can always be replaced by any $\mathcal{B}$ satisfying $|\mathcal{B}| \leq c|\nabla u|^{p-1}+d|u|^{p-1}+f$ with $c^{p}, d, f \in L^{n /(p-\varepsilon)}$ and $\varepsilon>0$. Also, the left-hand sides can be modified e.g. in the spirit of Example 3.4. Let us also mention that systems of diagonal type are often satisfied by steady state solutions of some reaction-diffusion systems.

Example 3.4. If $\nabla u^{j}$ and $u$ in (3.5) are replaced by e.g. $A_{j}(x) \nabla u^{j}$ and $C(x) u$, then the corresponding Euler-Lagrange system satisfies (3.1), provided that $A_{j}$ and $C$ are measurable matrices of types $n \times n$ and $N \times N$, such that $A_{j} \in L^{\infty}(\Omega)$, $C_{j} \in L^{n+\varepsilon}(\Omega)$ and $\left|A_{j}(x) \xi\right| \geq a_{0}|\xi|$ for some $a_{0}>0$, a.e. $x \in \Omega$ and all $\xi \in \mathbf{R}^{n}$.

Example 3.5. The functional

$$
u \longmapsto \int_{\Omega} \sum_{j=1}^{N}\left(a_{j}(x)|\nabla u|+b_{j}(x)\left|\nabla u^{j}\right|+c_{j}(x)|u|+d_{j}(x)\right)^{p} d x
$$

leads to the system (1.1) with

$$
\begin{aligned}
\mathcal{A}^{j}(x, u, \nabla u)= & \frac{b_{j} \nabla u^{j}}{\left|\nabla u^{j}\right|}\left(a_{j}|\nabla u|+b_{j}\left|\nabla u^{j}\right|+c_{j}|u|+d_{j}\right)^{p-1} \\
& +\sum_{i=1}^{N} \frac{a_{i} \nabla u^{j}}{|\nabla u|}\left(a_{i}|\nabla u|+b_{i}\left|\nabla u^{i}\right|+c_{i}|u|+d_{i}\right)^{p-1}, \\
\mathcal{B}(x, u, \nabla u)= & \sum_{i=1}^{N} \frac{c_{i} u^{i}}{|u|}\left(a_{i}|\nabla u|+b_{i}\left|\nabla u^{i}\right|+c_{i}|u|+d_{i}\right)^{p-1} .
\end{aligned}
$$


If all $a_{i}, b_{i}, c_{i}, d_{i}$ are nonnegative measurable functions such that $a_{i}, b_{i} \in L^{\infty}(\Omega)$, $c_{i}, d_{i} \in L^{n+\varepsilon}(\Omega)$ and $b_{i}(x) \geq b_{0}>0$ for all a.e. $x \in \Omega$, then the functions $\mathcal{A}^{j}$ and $\mathcal{B}^{j}$ satisfy the structural conditions (3.1). In fact, the left-hand sides $\mathcal{A}^{j}$ are a special case of the following general example.

Example 3.6. A large class of systems satisfying (3.1) is given by

$$
\mathcal{A}^{j}(q)=\left(\rho_{j}\left(\left|q^{j}\right|\right)+\sigma_{j}(x, u, q)\right) \frac{q^{j}}{\left|q^{j}\right|},
$$

where the functions $\rho_{j}$ and $\sigma_{j}$ are continuous in $u$ and $q$ (measurable in $x$ ) and satisfy $t^{p-1} \leq \rho_{j}(t) \leq a t^{p-1}$ and $0 \leq \sigma_{j}(x, u, q) \leq a|q|^{p-1}$, e.g. $\mathcal{A}^{j}(\nabla u)=$ $\left(\left|\nabla u^{j}\right|^{p-1}+|\nabla u|^{p-1}\right) \nabla u^{j} /\left|\nabla u^{j}\right|$.

\section{Some AUXILIARY ReSUlts}

In this section we present two lemmas which will be used in the proof of Theorem 3.2 As before we assume that $d \mu=w d x$, where $w$ is a $p$-admissible weight. The first lemma is a weighted modification and a slight improvement of Lemma 5.4 in Chapter 2 of Ladyzhenskaya-Ural'tseva [24] and provides us with a sufficient condition for the local boundedness of Sobolev functions.

Lemma 4.1. Let $U \in H^{1, p}\left(B\left(x_{0}, R\right), \mu\right), 1<p<\infty$, and assume that there exist constants $\gamma>0, k^{*} \geq 0$ and $0<\varepsilon<1-1 / \kappa$ such that for all $k \geq k^{*}$ and $\frac{1}{2} R<t<s<R$, the function $U$ satisfies the De Giorgi type inequality

$$
\int_{A(k, t)}|\nabla U|^{p} d \mu \leq \frac{\gamma}{(s-t)^{p}}\left(\int_{A(k, s)}(U-k)^{p} d \mu+k^{p} \frac{\mu(A(k, s))^{1 / \kappa+\varepsilon}}{\mu\left(B\left(x_{0}, R\right)\right)^{1 / \kappa+\varepsilon-1}}\right),
$$

where $A(k, s)=\left\{x \in B\left(x_{0}, s\right): U(x)>k\right\}$ and $\kappa$ is the constant from the weighted Sobolev inequality.

Then for all $k_{0} \geq k^{*}$,

$\operatorname{ess}^{\sup _{B\left(x_{0}, R / 2\right)} U}$

$$
\leq k_{0}+\max \left\{k_{0}, C(\gamma+1)^{1 / p \varepsilon}\left(\frac{1}{\mu\left(B\left(x_{0}, R\right)\right)} \int_{A\left(k_{0}, R\right)}\left(U-k_{0}\right)^{p} d \mu\right)^{1 / p}\right\},
$$

where the constant $C$ depends only on $p, \varepsilon$, the doubling constant $c_{1}$ of $\mu$ and the constants $c_{3}$ and $\kappa$ from the weighted Sobolev inequality.

Proof. Let $k_{0} \geq k^{*}$ and $B=B\left(x_{0}, R\right)$ be fixed. Put for $K \geq k_{0}$ and $j=0,1,2, \ldots$,

$$
\begin{aligned}
& r_{j}=\frac{1}{2}\left(1+2^{-j}\right) R, \\
& k_{j}=k_{0}+\left(1-2^{-j}\right) K, \\
& I_{j}=\int_{A\left(k_{j}, r_{j}\right)}\left(U-k_{j}\right)^{p} d \mu .
\end{aligned}
$$

We shall show that with a suitable choice of $K$,

$$
\int_{A\left(k_{0}+K, R / 2\right)}\left(U-k_{0}-K\right)^{p} d \mu=0,
$$

i.e. $U(x) \leq k_{0}+K$ for a.e. $x \in B\left(x_{0}, \frac{1}{2} R\right)$. Let $\zeta$ be a nonincreasing continuously differentiable function on $\mathbf{R}$ such that $\zeta(t)=1$ for $t \leq \frac{1}{2} R, \zeta(t)=0$ for $t \geq \frac{3}{4} R$ and $\left|\zeta^{\prime}(t)\right| \leq 8 / R$. Put for $j=0,1,2, \ldots$ and $x \in B$,

$$
\zeta_{j}(x)=\zeta\left(2^{j}\left(\left|x-x_{0}\right|-\frac{1}{2} R\right)\right) .
$$


Note that $\left|\nabla \zeta_{j}\right| \leq 2^{j+3} / R, \zeta_{j}=1$ on $B\left(x_{0}, r_{j}\right)$ and $\zeta_{j}=0$ outside $B\left(x_{0}, \bar{r}_{j}\right)$, where $\bar{r}_{j}=\frac{1}{2}\left(r_{j}+r_{j-1}\right)$. Hence, the function $\max \left\{U-k_{j}, 0\right\} \zeta_{j}$ belongs to $H_{0}^{1, p}\left(B\left(x_{0}, \bar{r}_{j}\right), \mu\right)$ and the Hölder inequality together with the weighted Sobolev inequality yields

$$
\begin{aligned}
I_{j} & \leq \int_{A\left(k_{j}, \bar{r}_{j}\right)}\left(U-k_{j}\right)^{p} \zeta_{j}^{p} d \mu \\
& \leq \mu\left(A\left(k_{j}, \bar{r}_{j}\right)\right)^{1-1 / \kappa}\left(\int_{A\left(k_{j}, \bar{r}_{j}\right)}\left(U-k_{j}\right)^{p \kappa} \zeta_{j}^{p \kappa} d \mu\right)^{1 / \kappa} \\
& \leq \frac{c_{3}^{p} \bar{r}_{j}^{p} \mu\left(A\left(k_{j}, \bar{r}_{j}\right)\right)^{1-1 / \kappa}}{\mu\left(B\left(x_{0}, \bar{r}_{j}\right)\right)^{1-1 / \kappa}} \int_{A\left(k_{j}, \bar{r}_{j}\right)}\left|\nabla\left(\left(U-k_{j}\right) \zeta_{j}\right)\right|^{p} d \mu \\
& \leq \frac{C R^{p} \mu\left(A\left(k_{j}, \bar{r}_{j}\right)\right)^{1-1 / \kappa}}{\mu\left(B\left(x_{0}, \bar{r}_{j}\right)\right)^{1-1 / \kappa}}\left(\int_{A\left(k_{j}, \bar{r}_{j}\right)}|\nabla U|^{p} d \mu+\frac{2^{p(j+3)}}{R^{p}} \int_{A\left(k_{j}, \bar{r}_{j}\right)}\left(U-k_{j}\right)^{p} d \mu\right),
\end{aligned}
$$

where $C=2^{p-1} c_{3}^{p}$.

The last integral on the right-hand side is not larger than $I_{j-1}$. The first integral on the right-hand side is estimated by means of the inequality (4.1) in the statement of the lemma with $t=\bar{r}_{j}$ and $s=r_{j-1}$ as follows:

$$
\begin{aligned}
\int_{A\left(k_{j}, \bar{r}_{j}\right)}|\nabla U|^{p} d \mu & \leq \frac{\gamma 2^{p(j+2)}}{R^{p}}\left(\int_{A\left(k_{j}, r_{j-1}\right)}\left(U-k_{j}\right)^{p} d \mu+k_{j}^{p} \frac{\mu\left(A\left(k_{j}, r_{j-1}\right)\right)^{1 / \kappa+\varepsilon}}{\mu(B)^{1 / \kappa+\varepsilon-1}}\right) \\
& \leq \frac{\gamma 2^{p(j+3)}}{R^{p}}\left(I_{j-1}+K^{p} \frac{\mu\left(A\left(k_{j}, r_{j-1}\right)\right)^{1 / \kappa+\varepsilon}}{\mu(B)^{1 / \kappa+\varepsilon-1}}\right) .
\end{aligned}
$$

Inserting this into (4.2) together with $A\left(k_{j}, \bar{r}_{j}\right) \subset A\left(k_{j}, r_{j-1}\right)$ and the doubling property of $\mu$ gives

$$
I_{j} \leq C 2^{p j}\left(\frac{\mu\left(A\left(k_{j}, r_{j-1}\right)\right)}{\mu(B)}\right)^{1-1 / \kappa}\left((\gamma+1) I_{j-1}+\gamma K^{p} \frac{\mu\left(A\left(k_{j}, r_{j-1}\right)\right)^{1 / \kappa+\varepsilon}}{\mu(B)^{1 / \kappa+\varepsilon-1}}\right),
$$

where $C$ depends only on $p, c_{1}, c_{3}$ and $\kappa$. Next, we need an upper bound for $\mu\left(A\left(k_{j}, r_{j-1}\right)\right)$. It is easily obtained from the inequalities

$$
I_{j-1} \geq \int_{A\left(k_{j}, r_{j-1}\right)}\left(U-k_{j-1}\right)^{p} d \mu \geq 2^{-p j} K^{p} \mu\left(A\left(k_{j}, r_{j-1}\right)\right),
$$

and the estimate (4.3) becomes

$$
\begin{aligned}
I_{j} & \leq C(\gamma+1) 2^{p j}\left(\frac{2^{p j} I_{j-1}}{K^{p} \mu(B)}\right)^{1-1 / \kappa}\left(I_{j-1}+K^{p}\left(\frac{2^{p j} I_{j-1}}{K^{p} \mu(B)}\right)^{1 / \kappa+\varepsilon} \mu(B)\right) \\
& \leq C(\gamma+1) 2^{p j(2+\varepsilon)} I_{j-1}\left(\left(\frac{I_{j-1}}{K^{p} \mu(B)}\right)^{1-1 / \kappa}+\left(\frac{I_{j-1}}{K^{p} \mu(B)}\right)^{\varepsilon}\right),
\end{aligned}
$$

where $C$ depends only on $p, c_{1}, c_{3}$ and $\kappa$. As $\varepsilon \leq 1-1 / \kappa$, we get for all $K \geq$ $\left(I_{0} / \mu(B)\right)^{1 / p} \geq\left(I_{j-1} / \mu(B)\right)^{1 / p}$,

$$
I_{j} \leq \frac{C(\gamma+1) \theta^{j}}{K^{p \varepsilon} \mu(B)^{\varepsilon}} I_{j-1}^{1+\varepsilon} \quad \text { for } j=1,2, \ldots,
$$


where $\theta=2^{p(2+\varepsilon)}$ and the constant $C$ depends on the same parameters as before. Finally, find the smallest $K \geq k_{0}$ such that

$$
I_{0} \leq \frac{K^{p} \mu(B)}{(C(\gamma+1))^{1 / \varepsilon} \theta^{1 / \varepsilon^{2}} \theta^{1 / \varepsilon}} .
$$

It is then easily shown by induction that

$$
\int_{A\left(k_{0}+K, R / 2\right)}\left(U-k_{0}-K\right)^{p} d \mu \leq I_{j} \leq \frac{K^{p} \mu(B)}{(C(\gamma+1))^{1 / \varepsilon} \theta^{1 / \varepsilon^{2}} \theta^{(j+1) / \varepsilon}} \rightarrow 0,
$$

as $j \rightarrow \infty$, i.e. $U(x) \leq k_{0}+K$ for a.e. $x \in B\left(x_{0}, \frac{1}{2} R\right)$.

Before stating the next lemma, let us show that on $B\left(x_{0}, R\right)$ the structure conditions (3.1) can be simplified by putting

$$
m=\left\|e^{p /(p-1)}+f\right\|_{L^{\tau}\left(B\left(x_{0}, R\right)\right)}^{1 / p}+\|f\|_{L^{\tau}\left(B\left(x_{0}, R\right)\right)}^{1 /(p-1)},
$$

where $\tau=\kappa /(\kappa-\varepsilon-1)$. Then the functions $\mathcal{A}^{j}$ and $\mathcal{B}^{j}$ satisfy

$$
\begin{aligned}
\left|\mathcal{A}^{j}(x, u, q)\right| & \leq\left(a|q|^{p-1}+\bar{b}(x)|\bar{u}|^{p-1}\right) w(x), \\
\left|\mathcal{B}^{j}(x, u, q)\right| & \leq\left(c(x)|q|^{p-1}+\bar{d}(x)|\bar{u}|^{p-1}\right) w(x), \\
\mathcal{A}^{j}(x, u, q) \cdot q^{j} & \geq\left(\left|q^{j}\right|^{p}-\bar{d}(x)|\bar{u}|^{p}\right) w(x),
\end{aligned}
$$

where $\bar{u}=|u|+m, \bar{b}=b+e / m^{p-1}$ and $\bar{d}=d+f / m^{p-1}+f / m^{p}$. The functions $\bar{b}^{p /(p-1)}$ and $\bar{d}$ obviously belong to $L^{\tau}\left(B\left(x_{0}, R\right), \mu\right)$ and their norms depend only on $p$ and the norms of $b^{p /(p-1)}$ and $d$.

The following lemma will be used for estimating the terms containing $|\nabla u|$.

Lemma 4.2. Let the assumptions of Theorem 3.2 be satisfied with $1<p<\infty$ and put

$$
U=\max \left\{\left|u^{1}\right|, \ldots,\left|u^{N}\right|\right\} .
$$

For $0<s<R$ and $k \geq m$ let also $A=\left\{x \in B\left(x_{0}, s\right): U(x)>k\right\}$ and $\omega=$ $\max \{U-k, 0\}$. Then there exists a positive constant $C_{0}$, depending only on $a, p$ and $N$, such that for all $k \geq m$ and all $\eta \in C_{0}^{\infty}\left(B\left(x_{0}, s\right)\right)$ satisfying $0 \leq \eta \leq 1$,

$$
\int_{A}\left(\frac{\omega}{U}\right)^{p}|\nabla u|^{p} \eta^{p} d \mu \leq C_{0}\left(\int_{A}|\nabla U|^{p} \eta^{p} d \mu+\int_{A} \omega^{p}|\nabla \eta|^{p} d \mu+\int_{A} g \bar{u}^{p} \eta^{p} d \mu\right),
$$

where $g=\bar{b}^{p /(p-1)}+c^{p}+\bar{d}$.

Proof. For $j=1, \ldots, N$, put

$$
\varphi^{j}=\left(\frac{\omega}{U}\right)^{p}\left(u^{j}-U\right) \eta^{p} \quad \text { and } \quad v^{j}=u^{j}-\varphi^{j}
$$

To be able to use $\varphi=\left(\varphi^{1}, \ldots, \varphi^{N}\right)$ and $v=\left(v^{1}, \ldots, v^{N}\right)$ as test functions in (3.2) and (3.3), we need to show that $\varphi \in H_{0}^{1, p}\left(B\left(x_{0}, R\right), \mu\right)^{N}$ and $v \in K$. We have $v^{j} \geq u^{j} \geq \psi_{j}$ and as $\eta \in C_{0}^{\infty}\left(B\left(x_{0}, s\right)\right)$, it is enough to show that $(\omega / U)^{p}\left(u^{j}-U\right) \in$ $H^{1, p}(\Omega, \mu)$. 
Write

$$
\left(\frac{\omega}{U}\right)^{p}\left(u^{j}-U\right)=\left(\frac{V-k}{V}\right)^{p}\left(u^{j}-V\right),
$$

where $V=\max \{U, k\}$, and let $V_{i}, v_{i} \in C^{\infty}(\Omega), i=1,2, \ldots$, be such that $V_{i} \rightarrow V$ and $v_{i} \rightarrow u^{j}$ both in $H^{1, p}(\Omega, \mu)$ and a.e. in $\Omega$. By (2.1) we may assume that $V_{i} \geq k$ and $\left|v_{i}\right| \leq V_{i}$ for all $i=1,2, \ldots$. A direct calculation shows that

$$
\begin{aligned}
&\left\|\left(\frac{V_{i}-k}{V_{i}}\right)^{p}\left(v_{i}-V_{i}\right)-\left(\frac{V-k}{V}\right)^{p}\left(u^{j}-V\right)\right\|_{H^{1, p}(\Omega, \mu)} \\
& \leq\left\|\left(\frac{V_{i}-k}{V_{i}}\right)^{p}\left(v_{i}-u^{j}+V-V_{i}\right)\right\|_{L^{p}(\Omega, \mu)} \\
&+\left\|\left(\left(\frac{V_{i}-k}{V_{i}}\right)^{p}-\left(\frac{V-k}{V}\right)^{p}\right)\left(u^{j}-V\right)\right\|_{L^{p}(\Omega, \mu)} \\
&+p k\left\|\left(\frac{V_{i}-k}{V_{i}}\right)^{p-1} \frac{v_{i}-V_{i}}{V_{i}^{2}}\left(\nabla V_{i}-\nabla V\right)\right\|_{L^{p}(\Omega, \mu)} \\
&+p k\left\|\left(\left(\frac{V_{i}-k}{V_{i}}\right)^{p-1} \frac{v_{i}-V_{i}}{V_{i}^{2}}-\left(\frac{V-k}{V}\right)^{p-1} \frac{u-V}{V^{2}}\right) \nabla V\right\|_{L^{p}(\Omega, \mu)} \\
&+\left\|\left(\frac{V_{i}-k}{V_{i}}\right)^{p}\left(\nabla v_{i}-\nabla u^{j}+\nabla V-\nabla V_{i}\right)\right\|_{L^{p}(\Omega, \mu)} \\
&+\left\|\left(\left(\frac{V_{i}-k}{V_{i}}\right)^{p}-\left(\frac{V-k}{V}\right)^{p}\right)\left(\nabla u^{j}-\nabla V\right)\right\|_{L^{p}(\Omega, \mu)} \\
&= I_{1}+\cdots+I_{6} .
\end{aligned}
$$

As $0 \leq\left(V_{i}-k\right) / V_{i} \leq 1,\left|v_{i}-V_{i}\right| \leq 2 V_{i}$ and $V_{i} \geq k$, the terms $I_{1}, I_{3}$ and $I_{5}$ can be estimated from above by the $H^{1, p}(\Omega, \mu)$-norms of $V_{i}-V$ and $v_{i}-u^{j}$ and tend to zero as $i \rightarrow \infty$. All the other terms tend to zero as $i \rightarrow \infty$ by the dominated convergence theorem. This shows that the function $(\omega / U)^{p}\left(u^{j}-k\right)$ belongs to $H^{1, p}(\Omega, \mu)$ and consequently $v \in K$.

Moreover, on $A$ we have

$$
\begin{aligned}
\nabla\left(u^{j}-v^{j}\right)= & \left(\frac{\omega}{U}\right)^{p} \nabla u^{j} \eta^{p}+\left(\frac{\omega}{U}\right)^{p-1}\left(\frac{p k\left(u^{j}-U\right)}{U^{2}}-\frac{\omega}{U}\right) \nabla U \eta^{p} \\
& +p\left(\frac{\omega}{U}\right)^{p}\left(u^{j}-U\right) \eta^{p-1} \nabla \eta .
\end{aligned}
$$

Note also that $\left|u^{j}-U\right| \leq 2 U, 0 \leq \omega \leq U \leq \bar{u}$, and $\left|p k\left(u^{j}-U\right) / U^{2}-\omega / U\right| \leq$ $2 p+1$ on $A$. As $u-v=0$ outside $A$, inserting $\varphi$ and $v$ into (3.2) and (3.3), respectively, together with the structure conditions (4.4) and some straightforward 
simplifications gives

$$
\begin{aligned}
& \sum_{j=1}^{N} \int_{A}\left(\frac{\omega}{U}\right)^{p}\left|\nabla u^{j}\right|^{p} \eta^{p} d \mu \\
& \leq N(2 p+1) \int_{A} a\left(\frac{\omega}{U}\right)^{p-1}|\nabla U||\nabla u|^{p-1} \eta^{p} d \mu+N(2 p+1) \int_{A} \bar{b}|\nabla U| \bar{u}^{p-1} \eta^{p} d \mu \\
&+2 N p \int_{A} a\left(\frac{\omega}{U}\right)^{p-1} \omega|\nabla u|^{p-1} \eta^{p-1}|\nabla \eta| d \mu+2 N p \int_{A} \bar{b} \omega \bar{u}^{p-1} \eta^{p-1}|\nabla \eta| d \mu \\
&+2 N \int_{A} c\left(\frac{\omega}{U}\right)^{p-1} \bar{u}|\nabla u|^{p-1} \eta^{p} d \mu+3 N \int_{A} \bar{d} \bar{u}^{p} \eta^{p} d \mu \\
& \leq N(2 p+1)\left(I_{1}+\cdots+I_{6}\right) .
\end{aligned}
$$

The integrals on the right-hand side will be estimated by means of the Young inequality. By $\nu$ we denote a small positive number which will be chosen later. We get

$$
\begin{aligned}
& I_{1} \leq \frac{\nu}{p^{\prime}} \int_{A}\left(\frac{\omega}{U}\right)^{p}|\nabla u|^{p} \eta^{p} d \mu+\frac{a^{p}}{p \nu^{p-1}} \int_{A}|\nabla U|^{p} \eta^{p} d \mu, \\
& I_{2} \leq \frac{1}{p^{\prime}} \int_{A} \bar{b}^{p /(p-1)} \bar{u}^{p} \eta^{p} d \mu+\frac{1}{p} \int_{A}|\nabla U|^{p} \eta^{p} d \mu, \\
& I_{3} \leq \frac{\nu}{p^{\prime}} \int_{A}\left(\frac{\omega}{U}\right)^{p}|\nabla u|^{p} \eta^{p} d \mu+\frac{a^{p}}{p \nu^{p-1}} \int_{A} \omega^{p}|\nabla \eta|^{p} d \mu, \\
& I_{4} \leq \frac{1}{p^{\prime}} \int_{A} \bar{b}^{p /(p-1)} \bar{u}^{p} \eta^{p} d \mu+\frac{1}{p} \int_{A} \omega^{p}|\nabla \eta|^{p} d \mu, \\
& I_{5} \leq \frac{\nu}{p^{\prime}} \int_{A}\left(\frac{\omega}{U}\right)^{p}|\nabla u|^{p} \eta^{p} d \mu+\frac{1}{p \nu^{p-1}} \int_{A} c^{p} \bar{u}^{p} \eta^{p} d \mu,
\end{aligned}
$$

where $p^{\prime}=p /(p-1)$. Inserting the above estimates into (4.5) we obtain

$$
\begin{aligned}
\int_{A}\left(\frac{\omega}{U}\right)^{p}|\nabla u|^{p} \eta^{p} d \mu \leq & \frac{3 N(2 p+1) \nu}{p^{\prime}} \int_{A}\left(\frac{\omega}{U}\right)^{p}|\nabla u|^{p} \eta^{p} d \mu \\
& +C\left(\int_{A}|\nabla U|^{p} \eta^{p} d \mu+\int_{A} \omega^{p}|\nabla \eta|^{p} d \mu+\int_{A} g \bar{u}^{p} \eta^{p} d \mu\right),
\end{aligned}
$$

where $g=\bar{b}^{p /(p-1)}+c^{p}+\bar{d}$ and the constant $C$ depends only on $a, p, N$ and $\nu$.

To finish the proof, choose $\nu=p^{\prime} / 6 N(2 p+1)$, then the first integral on the right-hand side is absorbed by the left-hand side and the lemma follows.

Remark 4.3. For systems of equations, the proof could be slightly simplified by putting $\varphi^{j}=(\omega / U)^{p} u^{j} \eta^{p}$. It would also give a better constant $C_{0}$. In the proof, we have chosen $\varphi^{j}$ which work both for equations and variational inequalities.

\section{LOCAL BOUNDEDNESS OF WEAK SOLUTIONS}

Proof of Theorem 3.2. We shall treat the cases (a) and (b) simultaneously and show that the function $U=\max \left\{\left|u^{1}\right|, \ldots,\left|u^{N}\right|\right\}$ is bounded on $B\left(x_{0}, \frac{1}{2} R\right)$. The proof is based on Lemma 4.1, so we need to estimate $\int_{A(k, t)}|\nabla U|^{p} d \mu$, where $A(k, t)=\{x \in$ $\left.B\left(x_{0}, t\right): U(x)>k\right\}$. 
Let $B=B\left(x_{0}, R\right)$ and $k \geq k_{0}=\max \left\{m, \operatorname{ess} \sup _{B\left(x_{0}, R\right)} \psi^{j}, j=1, \ldots, N\right\}$ be fixed and put for $\frac{1}{2} R<t<s<R$ and $j=1, \ldots, N$,

$$
\begin{aligned}
\omega & =\max \{U-k, 0\}, \\
\omega^{j} & =\max \left\{\left|u^{j}\right|-k, 0\right\}, \\
A & =\left\{x \in B\left(x_{0}, s\right): U(x)>k\right\}=A(k, s), \\
A^{j} & =\left\{x \in B\left(x_{0}, s\right):\left|u^{j}(x)\right|>k\right\}, \\
\varphi^{j} & =\omega^{j} \eta^{p} \operatorname{sign} u^{j}, \\
v^{j} & =u^{j}-\varphi^{j},
\end{aligned}
$$

where $\eta \in C_{0}^{\infty}\left(B\left(x_{0}, s\right)\right), 0 \leq \eta \leq 1, \eta=1$ on $B\left(x_{0}, t\right)$ and $|\nabla \eta| \leq 2 /(s-t)$. It is easily verified that $u^{j}-v^{j}=\varphi^{j} \in H_{0}^{1, p}\left(B\left(x_{0}, R\right)\right)$ and moreover,

$$
\begin{array}{ll}
v^{j}(x)=u^{j}(x) \geq \psi^{j}(x) & \text { if } \omega^{j}(x)=0, \\
v^{j}(x) \geq u^{j}(x) \geq \psi^{j}(x) & \text { if } \omega^{j}(x)>0 \text { and } \operatorname{sign} u^{j}(x)=-1 \\
v^{j}(x)=\left(1-\eta^{p}\right) u^{j}(x)+\eta^{p} k \geq \psi^{j}(x) & \text { if } \omega^{j}(x)>0 \text { and } \operatorname{sign} u^{j}(x)=1 .
\end{array}
$$

Hence $v=\left(v^{1}, \ldots, v^{N}\right) \in K$ and on $A^{j}$ we have

$$
\nabla\left(u^{j}-v^{j}\right)=\nabla \varphi^{j}=\nabla u^{j} \eta^{p}+p \omega^{j} \eta^{p-1} \nabla \eta \operatorname{sign} u^{j} .
$$

Inserting $\varphi=\left(\varphi^{1}, \ldots, \varphi^{N}\right)$ and $v$ into (3.2) and (3.3), respectively, together with the structure conditions (4.4), $\omega^{j} \leq \omega \leq \bar{u}$ and $A^{j} \subset A$ gives

$$
\begin{aligned}
\sum_{j=1}^{N} \int_{A^{j}}\left|\nabla u^{j}\right|^{p} \eta^{p} d \mu \\
\leq N p \int_{A} a \omega|\nabla u|^{p-1} \eta^{p-1}|\nabla \eta| d \mu+N p \int_{A} \bar{b} \omega \bar{u}^{p-1} \eta^{p-1}|\nabla \eta| d \mu \\
\quad+N \int_{A} c \omega|\nabla u|^{p-1} \eta^{p} d \mu+2 N \int_{A} \bar{d} \bar{u}^{p} \eta^{p} d \mu \\
=I_{1}+I_{2}+I_{3}+I_{4} .
\end{aligned}
$$

We shall first simplify the left-hand side. Note that $A=\bigcup_{j=1}^{N} A^{j}$ and that for a.e. $x$ we have $|\nabla U(x)|=\left|\nabla u^{j}(x)\right|$ whenever $U(x)=\left|u^{j}(x)\right|$. Also if $x \in A$ and $U(x)=\left|u^{j}(x)\right|$, then $\left|u^{j}(x)\right|>k$, i.e. $x \in A^{j}$. Hence

$$
\int_{A}|\nabla U|^{p} \eta^{p} d \mu \leq \sum_{j=1}^{N} \int_{\left\{x \in A: U(x)=\left|u^{j}(x)\right|\right\}}\left|\nabla u^{j}\right|^{p} \eta^{p} d \mu \leq \sum_{j=1}^{N} \int_{A^{j}}\left|\nabla u^{j}\right|^{p} \eta^{p} d \mu .
$$

The term $I_{2}$ in (5.1) is estimated using the Young inequality as follows

$$
I_{2} \leq N \int_{A} \omega^{p}|\nabla \eta|^{p} d \mu+N(p-1) \int_{A} \bar{b}^{p /(p-1)} \bar{u}^{p} \eta^{p} d \mu .
$$

To estimate $I_{1}$ and $I_{3}$, let $\nu=p / 2\left(p^{2}-1\right) N C_{0}$, where $C_{0}$ is the constant from Lemma 4.2. Again by the Young inequality and using that $U=\omega+k \leq \bar{u}$ we 
obtain,

$$
\begin{aligned}
& I_{1} \leq N(p-1) \nu \int_{A}\left(\frac{\omega}{U}\right)^{p /(p-1)}|\nabla u|^{p} \eta^{p} d \mu+\frac{N a^{p}}{\nu^{p-1}} \int_{A} 2^{p-1}\left(\omega^{p}+k^{p}\right)|\nabla \eta|^{p} d \mu, \\
& I_{3} \leq \frac{N(p-1) \nu}{p} \int_{A}\left(\frac{\omega}{U}\right)^{p /(p-1)}|\nabla u|^{p} \eta^{p} d \mu+\frac{N}{p \nu^{p-1}} \int_{A} c^{p} \bar{u}^{p} \eta^{p} d \mu .
\end{aligned}
$$

As $p \leq 2$, we have $p /(p-1) \geq p$ and thus $(\omega / U)^{p /(p-1)} \leq(\omega / U)^{p}$. It follows that the first integrals on the right-hand sides in (5.4) can be estimated by Lemma 4.2 This, together with the estimates (5.1), (5.2) and (5.3) yields

$$
\begin{aligned}
& \int_{A}|\nabla U|^{p} \eta^{p} d \mu \leq \frac{1}{2} \int_{A}|\nabla U|^{p} \eta^{p} d \mu \\
& \quad+C\left(\int_{A} \omega^{p}|\nabla \eta|^{p} d \mu+k^{p} \int_{A}|\nabla \eta|^{p} d \mu+\int_{A} g \bar{u}^{p} \eta^{p} d \mu\right),
\end{aligned}
$$

where $g=\bar{b}^{p /(p-1)}+c^{p}+\bar{d}$ and $C$ is a constant depending only on $a, p$ and $N$. Note that the first term on the right-hand side is absorbed by the left-hand side.

To be able to apply Lemma 4.1 we need to estimate the last two integrals in (5.5). The function $g$ belongs to $L^{\tau}\left(B\left(x_{0}, R\right), \mu\right)$, with $\tau=\kappa /(\kappa-1-\varepsilon)$ and its norm depends only on $p$ and the norms of $b^{p /(p-1)}, c^{p}$ and $d$. As $k \geq m$, we have on $A$,

$$
\bar{u}^{p}=(|u|+m)^{p} \leq N^{p}(U+m)^{p} \leq N^{p}(\omega+k+m)^{p} \leq 2^{p-1} N^{p} \omega^{p}+2^{2 p-1} N^{p} k^{p} .
$$

Thus, the last integral in (5.5) can be estimated using a sum of two integrals, which in turn are estimated by means of the Hölder and Sobolev inequalities and the doubling property of $\mu$ as follows

$$
\begin{aligned}
\int_{A} g \omega^{p} \eta^{p} d \mu & \leq\left(\int_{A}(\omega \eta)^{p \kappa} d \mu\right)^{1 / \kappa} \mu(A)^{\varepsilon / \kappa}\left(\int_{B} g^{\tau} d \mu\right)^{1 / \tau} \\
& \leq \frac{c_{1} c_{3}^{p} R^{p}\|g\| \mu(A)^{\varepsilon / \kappa}}{\mu(B)^{\varepsilon / \kappa}} \int_{A} \mid \nabla(\omega \eta)^{p} d \mu, \\
k^{p} \int_{A} g \eta^{p} d \mu & \leq \frac{k^{p}\|g\| \mu(A)^{(1+\varepsilon) / \kappa}}{\mu(B)^{(1+\varepsilon) / \kappa-1}},
\end{aligned}
$$

so that the last term in (5.5) becomes

$$
\begin{aligned}
C \int_{A} g \bar{u}^{p} \eta^{p} d \mu \leq & \frac{C\|g\| \mu(A)^{\varepsilon / \kappa}}{\mu(B)^{\varepsilon / \kappa}}\left(\int_{A}|\nabla U|^{p} \eta^{p} d \mu+\int_{A} \omega^{p}|\nabla \eta|^{p} d \mu\right) \\
& +\frac{C k^{p}\|g\| \mu(A)^{(1+\varepsilon) / \kappa}}{\mu(B)^{(1+\varepsilon) / \kappa-1}}
\end{aligned}
$$

where $\|g\|=\left(f_{B} g^{\tau} d \mu\right)^{1 / \tau}, \tau=\kappa /(\kappa-1-\varepsilon)$, and the constant $C$ depends only on $p, N, a, c_{1}, c_{3}$ and $\kappa$. Next, as $\mu(A) \leq k^{-p} \int_{B} U^{p} d \mu$, we see that for

$$
k \geq k^{*}=C\|g\|^{\kappa / p \varepsilon}\left(f_{B} U^{p} d \mu\right)^{1 / p},
$$

the coefficient at $\int_{A}|\nabla U|^{p} \eta^{p} d \mu$ in (5.6) is at most $\frac{1}{4}$, i.e. that term is absorbed by the left-hand side of (5.5). Note that the constant $C$ in (5.7) depends only on $p$, $N, a, \varepsilon, c_{1}, c_{3}$ and $\kappa$. 
Also, as $|\nabla \eta| \leq 2 /(s-t)$ and $(1+\varepsilon) / \kappa<1$, we have

$$
k^{p} \int_{A}|\nabla \eta|^{p} d \mu \leq \frac{2^{p} k^{p}}{(s-t)^{p}} \mu(A) \leq \frac{2^{p} k^{p}}{(s-t)^{p}} \frac{\mu(A)^{(1+\varepsilon) / \kappa}}{\mu(B)^{(1+\varepsilon) / \kappa-1}} .
$$

Finally, as $\eta=1$ on $B\left(x_{0}, t\right)$, we get (with a new constant $C$ depending only on $p, N, a, \varepsilon, c_{1}, c_{3}$ and $\left.\kappa\right)$ for all $k \geq \max \left\{m, k^{*}, \operatorname{ess}_{\sup _{B}} \psi^{j}, j=1, \ldots, N\right\}$ and $\frac{1}{2} R<t<s<R$,

$$
\frac{1}{4} \int_{A(k, t)}|\nabla U|^{p} d \mu \leq \frac{C(\|g\|+1)}{(s-t)^{p}}\left(\int_{A} \omega^{p} d \mu+k^{p} \frac{\mu(A)^{(1+\varepsilon) / \kappa}}{\mu(B)^{(1+\varepsilon) / \kappa-1}}\right) .
$$

Lemma 4.1 with $k_{0}=\max \left\{m, k^{*}\right.$, $\left.\operatorname{ess}_{\sup _{B}} \psi^{j}, j=1, \ldots, N\right\}$ and $\varepsilon$ replaced by $\varepsilon / \kappa$ now finishes the proof.

Remark 5.1. Note that in the case of one equation, we have $U=|u|$ and $|\nabla u|=$ $|\nabla U|$, so that the estimates (5.4) and Lemma 4.2 are not needed and we can drop the assumption $p \leq 2$ in the statement of Theorem 3.2.

\section{Pointwise Differentiability of Weak Solutions}

In this section we shall extend the Reshetnyak-Hajłasz-Strzelecki method mentioned in the introduction to the weighted situation as well as to systems of equations and variational inequalities and show that the solutions are differentiable a.e. The idea of the method is as follows.

Definition 6.1. Let $x_{0} \in \Omega$ and $0<h<\operatorname{dist}\left(x_{0}, \partial \Omega\right)$. For a function $u: \Omega \rightarrow \mathbf{R}^{N}$ and $X \in B(0,1)$ we define the difference quotient $u_{h}$ of $u$ at $x_{0}$ by

$$
u_{h}(X)=\frac{u\left(x_{0}+h X\right)-u\left(x_{0}\right)}{h} .
$$

It is shown that the difference quotients $u_{h}$ of $u$ at $x_{0}$ satisfy equations similar to the original one and are thus bounded on $B\left(0, \frac{1}{2}\right)$ by constants depending on the parameters of the original equation and on $\left\|u_{h}\right\|_{L^{p}(B(0,1))}$. A standard theorem about $L^{p}$-differentials of Sobolev functions (e.g. Theorem 3.4.2 in Ziemer [39]) yields

$$
\left\|u_{h}\right\|_{L^{p}(B(0,1))} \rightarrow\left\|\nabla u\left(x_{0}\right) \cdot X\right\|_{L^{p}(B(0,1))}, \quad \text { as } h \rightarrow 0,
$$

and hence $\left\|u_{h}\right\|_{L^{p}(B(0,1))} \leq Q$ for sufficiently small $h$. The differentiability of $u$ then follows from the following theorem due to Stepanov (see [35] or Theorem 3.1.9 in Federer [6]).

Theorem 6.2. For a function $u: \Omega \rightarrow \mathbf{R}^{N}$ put

$$
A=\left\{x \in \Omega: \limsup _{y \rightarrow x} \frac{|u(y)-u(x)|}{|y-x|}<\infty\right\} .
$$

Then $A$ is Lebesgue measurable and $u$ is differentiable a.e. in A.

In order to extend this method to our situation, there are some difficulties to overcome, mainly due to the presence of the weight $w$. First, the difference quotients $u_{h}$ do not in general belong to $H^{1, p}(B(0,1), \mu)$, hence it is necessary to consider weights $w_{h}$ which vary as $h \rightarrow 0$. Second, in order to prove that the difference quotients $u_{h}$ of $u$ are bounded by a constant independent of $h$, we need a weighted version of (6.1), viz. Theorem 6.5 and Corollary 6.6 Finally, for variational inequalities, the obstacle $\psi$ has to be taken into account. 
Let us begin by the following lemma which shows that for sufficiently small $h$ the difference quotients of solutions of the systems (3.2) and (3.3) are solutions of systems similar to (3.2) and (3.3).

Lemma 6.3. Let $w$ be a p-admissible weight with $1<p<\infty$, and for a fixed $x_{0} \in \Omega$ and $0<h<\operatorname{dist}\left(x_{0}, \partial \Omega\right)$ let $\mu_{h}$ be the measure whose Radon-Nikodym derivative is $w_{h}(X)=w\left(x_{0}+h X\right)$, i.e. $d \mu_{h}=w_{h} d x$. Then the weight $w_{h}$ is $p$ admissible with the same constants $c_{1}$ and $c_{2}$ as $w$ and the difference quotients $u_{h}$ of $u$ at $x_{0}$ satisfy:

(a) If $u \in H^{1, p}(\Omega, \mu)^{N}$ is a weak solution of the system (3.2), then $u_{h} \in$ $H^{1, p}\left(B(0,1), \mu_{h}\right)^{N}$ and $u_{h}$ is a weak solution of the system

$$
\operatorname{div}\left(\mathcal{A}_{h}^{j}\left(X, u_{h}, \nabla u_{h}\right)\right)=\mathcal{B}_{h}^{j}\left(X, u_{h}, \nabla u_{h}\right), \quad j=1, \ldots, N .
$$

(b) If $u \in K$ is a solution of the obstacle problem (3.3), then $u_{h} \in K_{h}$, where

$$
\begin{array}{r}
K_{h}=\left\{v \in H^{1, p}\left(B(0,1), \mu_{h}\right)^{N}: v-u_{h} \in H_{0}^{1, p}\left(B(0,1), \mu_{h}\right)^{N}\right. \\
\text { and } \left.v \geq \psi_{h} \text { a.e. in } B(0,1)\right\}
\end{array}
$$

with $\psi_{h}(X)=\left(\psi\left(x_{0}+h X\right)-u\left(x_{0}\right)\right) / h$ and $u_{h}$ is a solution of the obstacle problem

$$
\begin{aligned}
& \sum_{j=1}^{N} \int_{B(0,1)} \mathcal{A}_{h}^{j}\left(X, u_{h}, \nabla u_{h}\right) \cdot \nabla\left(u_{h}^{j}-v^{j}\right) d X \\
& \quad+\sum_{j=1}^{N} \int_{B(0,1)} \mathcal{B}_{h}^{j}\left(X, u_{h}, \nabla u_{h}\right)\left(u_{h}^{j}-v^{j}\right) d X \leq 0
\end{aligned}
$$

for all $v \in K_{h}$.

Here, the functions $\mathcal{A}_{h}^{j}$ and $\mathcal{B}_{h}^{j}$ are defined by

$$
\begin{aligned}
& \mathcal{A}_{h}^{j}(X, v, q)=\mathcal{A}^{j}\left(x_{0}+h X, u\left(x_{0}\right)+h v, q\right), \\
& \mathcal{B}_{h}^{j}(X, v, q)=h \mathcal{B}^{j}\left(x_{0}+h X, u\left(x_{0}\right)+h v, q\right) .
\end{aligned}
$$

Proof. It is easily verified that the weights $w_{h}$ are $p$-admissible with the same constants $c_{1}$ and $c_{2}$ as $w$.

(a) The lemma follows immediately from (3.2) by the change of variables $x=$ $x_{0}+h X$.

(b) Let $v \in K_{h}$ and extend $v-u_{h}$ by zero outside $B(0,1)$. Put for $x \in \Omega$,

$$
\tilde{v}(x)=u(x)+h\left(v\left(\frac{x-x_{0}}{h}\right)-u_{h}\left(\frac{x-x_{0}}{h}\right)\right) .
$$

Then $\tilde{v}(x) \geq u(x)+(\psi(x)-u(x))=\psi(x)$ and $\tilde{v}=u$ outside $B\left(x_{0}, h\right)$, hence $\tilde{v} \in K$. Inserting $\tilde{v}$ into (3.3) and using the change of variables $x=x_{0}+h X$ yields (6.3).

In the rest of this section we shall frequently use the following Lebesgue differentiation theorem, see e.g. p. 14 in Ziemer [39] for the case $p=1$. The case $p>1$ then follows from the formula $|a-b|^{p} \leq\left|a^{p}-b^{p}\right|$ for $a, b \geq 0$.

Theorem 6.4. If $f \in L_{\mathrm{loc}}^{p}\left(\mathbf{R}^{n}, \mu\right)$, where $\mu$ is a Radon measure and $1 \leq p<\infty$, then $\mu$-a.e. $x$ is an $L^{p}(\mu)$-Lebesgue point of $f$, i.e. for $\mu$-a.e. $x \in \mathbf{R}^{n}$,

$$
\lim _{r \rightarrow 0}\left(f_{B(x, r)}|f(y)-f(x)|^{p} d \mu(y)\right)^{1 / p}=0 .
$$


The following theorem is a weighted generalization of a classical result about $L^{p}$-differentials, for a proof see the author's paper [2].

Theorem 6.5. Let $w$ be a p-admissible weight, $1<p<\infty$. Let $d \mu=w d x$ and $u \in H^{1, p}(\Omega, \mu)$. Then for a.e. $x_{0} \in \Omega$,

$$
\lim _{h \rightarrow 0} \frac{1}{h}\left(f_{B\left(x_{0}, h\right)}\left|u(x)-u\left(x_{0}\right)-\nabla u\left(x_{0}\right) \cdot\left(x-x_{0}\right)\right|^{p} d \mu(x)\right)^{1 / p}=0 .
$$

Corollary 6.6. If $w$ is a p-admissible weight, $1<p<\infty$, and $u \in H^{1, p}(\Omega, \mu)$, then for a.e. $x_{0} \in \Omega$,

$$
\lim _{h \rightarrow 0}\left(f_{B(0,1)}\left|u_{h}\right|^{p} d \mu_{h}\right)^{1 / p}=\left(f_{B(0,1)}\left|\nabla u\left(x_{0}\right) \cdot X\right|^{p} d X\right)^{1 / p} .
$$

Proof. The triangle and the Minkowski inequalities show that

$$
\begin{aligned}
\mid\left(\int_{B(0,1)}\left|u_{h}\right|^{p} d \mu_{h}\right)^{1 / p} & -\left(w\left(x_{0}\right) \int_{B(0,1)}\left|\nabla u\left(x_{0}\right) \cdot X\right|^{p} d X\right)^{1 / p} \mid \\
& \leq\left\|u_{h}-\nabla u\left(x_{0}\right) \cdot X\right\|_{L^{p}\left(B(0,1), \mu_{h}\right)} \\
& +\left\|\nabla u\left(x_{0}\right) \cdot X\left(w_{h}(X)^{1 / p}-w\left(x_{0}\right)^{1 / p}\right)\right\|_{L^{p}(B(0,1))} .
\end{aligned}
$$

The first term on the right-hand side tends to zero, as $h \rightarrow 0$, for a.e. $x_{0} \in \Omega$ by Theorem 6.5 and the fact that for a.e. $x_{0}, \mu_{h}(B(0,1)) \rightarrow w\left(x_{0}\right)|B(0,1)|$, as $h \rightarrow 0$. The last term on the right-hand side is not larger than $\left|\nabla u\left(x_{0}\right)\right| \| w_{h}(X)^{1 / p}-$ $w\left(x_{0}\right)^{1 / p} \|_{L^{p}(B(0,1))}$ and tends to zero, as $h \rightarrow 0$, whenever $x_{0}$ is an $L^{p}$-Lebesgue point of $w^{1 / p}$. The fact that for a.e. $x_{0}, \mu_{h}(B(0,1)) \rightarrow w\left(x_{0}\right)|B(0,1)|$, as $h \rightarrow 0$, finishes the proof.

We are now ready to prove the main result of this section - the differentiability a.e. of weak solutions of the systems (3.2) and (3.3).

Theorem 6.7. Suppose that the structure conditions (3.1) are satisfied with a $p$ admissible weight $w, 1<p \leq 2$. Let $d \mu=w d x$ and consider one of the following two cases:

(a) $u \in H^{1, p}(\Omega, \mu)^{N}$ is a weak solution of the system (3.2), or

(b) $u \in K$ is a solution of the obstacle problem (3.3) and assume that $\psi$ is differentiable at a.e. $x \in \Omega$.

Then any representative of $u$ satisfying for all $x \in \Omega$ and $j=1, \ldots, N$,

$$
\liminf _{r \rightarrow 0} f_{B(x, r)} u^{j} d \mu \leq u^{j}(x) \leq \limsup _{r \rightarrow 0} f_{B(x, r)} u^{j} d \mu,
$$

is differentiable a.e. in $\Omega$ in the classical sense.

Remark 6.8. The proof actually shows that even if $\psi$ is not differentiable a.e., then the solution $u$ is differentiable a.e. on the set where $\psi$ is differentiable. Regarding condition (6.4), recall that by Theorem 6.4 all $u \in L^{p}(\Omega, \mu)$ can be modified on a set of measure zero, so that $u(x)=\limsup _{r \rightarrow 0} f_{B(x, r)} u d \mu$ holds for all $x \in \Omega$.

Proof. Let $x_{0} \in \Omega$ be such that the conclusion of Corollary 6.6 holds at $x_{0}$ and assume that $\psi$ is differentiable at $x_{0}$. Let also $x_{0}$ be an $L^{1}$-Lebesgue point of $w$ and an $L^{\tau}(\mu)$-Lebesgue point of the functions $b^{p /(p-1)}, c^{p}, d, e^{p /(p-1)}$ and $f$, where $\tau=\kappa /(\kappa-1-\varepsilon)$. By Theorem 6.4, almost every point of $\Omega$ has this property. 
For $0<h<\operatorname{dist}\left(x_{0}, \partial \Omega\right)$ consider the difference quotients

$$
u_{h}(X)=\left(u\left(x_{0}+h X\right)-u\left(x_{0}\right)\right) / h, \quad X \in B(0,1) .
$$

If we can show that for sufficiently small $h$, the difference quotients $u_{h}$ are bounded on $B\left(0, \frac{1}{2}\right)$ by a constant independent of $h$, then Theorem 6.2 implies that $u$ is differentiable a.e. in $\Omega$.

The uniform boundedness of the difference quotients will be proved by means of Lemma 6.3 and Theorem 3.2. Lemma 6.3 yields that for $0<h<\operatorname{dist}\left(x_{0}, \partial \Omega\right)$ the difference quotients $u_{h}$ are solutions of (3.2) and (3.3) with $\mathcal{A}^{j}$ and $\mathcal{B}^{j}$ replaced by $\mathcal{A}_{h}^{j}$ and $\mathcal{B}_{h}^{j}$, respectively. Next, we verify that the functions $\mathcal{A}_{h}^{j}$ and $\mathcal{B}_{h}^{j}$ satisfy the assumptions of Theorem 3.2. We can assume that $h \leq 1$. A direct calculation shows that for a.e. $X \in B(0,1)$, all $v \in \mathbf{R}^{n}$ and all $q \in \mathbf{R}^{n N}$,

$$
\begin{aligned}
\left|\mathcal{A}_{h}^{j}(X, v, q)\right| & \leq\left(a|q|^{p-1}+b_{h}(X)|v|^{p-1}+e_{h}(X)\right) w_{h}(X), \\
\left|\mathcal{B}_{h}^{j}(X, v, q)\right| & \leq\left(c_{h}(X)|q|^{p-1}+d_{h}(X)|v|^{p-1}+f_{h}(X)\right) w_{h}(X), \\
\mathcal{A}_{h}^{j}(X, v, q) \cdot q^{j} & \geq\left(\left|q^{j}\right|^{p}-d_{h}(X)|v|^{p}-f_{h}(X)\right) w_{h}(X),
\end{aligned}
$$

where the weights $w_{h}$ are as in Lemma 6.3 and

$$
\begin{aligned}
& b_{h}(X)=h^{p-1} b\left(x_{0}+h X\right), \\
& c_{h}(X)=h c\left(x_{0}+h X\right), \\
& d_{h}(X)=2^{p-1} h^{p} d\left(x_{0}+h X\right), \\
& e_{h}(X)=e\left(x_{0}+h X\right)+b\left(x_{0}+h X\right)\left|u\left(x_{0}\right)\right|^{p-1}, \\
& f_{h}(X)=f\left(x_{0}+h X\right)+2^{p-1} d\left(x_{0}+h X\right)\left|u\left(x_{0}\right)\right|^{p-1} .
\end{aligned}
$$

Theorem 3.2 now yields

$$
\operatorname{ess}_{\sup _{B(0,1 / 2)}}\left|u_{h}\right| \leq 2\left(\Psi_{h}+m_{h}\right)+C_{h}\left(f_{B(0,1)}\left|u_{h}\right|^{p} d \mu_{h}\right)^{1 / p}
$$

where $\Psi_{h}, m_{h}$ and $C_{h}$ are as in Theorem 3.2 with $\psi^{j}, b, c, d, e$ and $f$ replaced by $\psi_{h}^{j}, b_{h}, c_{h}, d_{h}, e_{h}$ and $f_{h}$. As $x_{0}$ is a Lebesgue point of $b^{p /(p-1)}, c^{p}, d, e^{p /(p-1)}$ and $f$, we get that $C_{h} \rightarrow C^{\prime}$, as $h \rightarrow 0$, and for sufficiently small $h$ the constant $m_{h}$ is bounded by a constant independent of $h$. To estimate $\Psi_{h}$, we need to distinguish between two cases:

(i) If $u^{j}\left(x_{0}\right)=\psi^{j}\left(x_{0}\right)$, then as $\psi^{j}$ is differentiable at $x_{0}$, we have

$$
\lim _{h \rightarrow 0} \psi_{h}^{j}(X)=\lim _{h \rightarrow 0} \frac{\psi^{j}\left(x_{0}+h X\right)-\psi^{j}\left(x_{0}\right)}{h}=\nabla \psi^{j}\left(x_{0}\right) \cdot X
$$

uniformly for $X \in B(0,1)$. If follows that for $0<h<h_{0}$ and sufficiently small $h_{0}$,

$$
\operatorname{ess}_{\sup } \sup _{B(1)} \psi_{h}^{j} \leq\left|\nabla \psi^{j}\left(x_{0}\right)\right|+1 \text {. }
$$

(ii) If $u^{j}\left(x_{0}\right)>\psi^{j}\left(x_{0}\right)$, then as $\psi^{j}$ is continuous at $x_{0}$, we have $u^{j}\left(x_{0}\right)>$ $\psi^{j}\left(x_{0}+h X\right)$ for all $X \in B(0,1), 0<h<h_{0}$ and sufficiently small $h_{0}$. This yields $\psi_{h}^{j}(X)=\left(\psi^{j}\left(x_{0}+h X\right)-u^{j}\left(x_{0}\right)\right) / h<0$ for all $X \in B(0,1)$.

Finally, Corollary [6.6] implies that $\lim _{h \rightarrow 0}\left(f_{B(0,1)}\left|u_{h}\right|^{p} d \mu_{h}\right)^{1 / p}<\infty$ and conse-

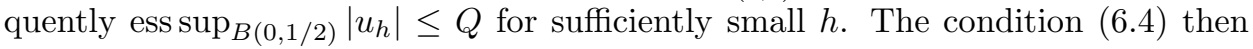
implies $\sup _{B(0,1 / 2)}\left|u_{h}\right| \leq Q$ and hence

$$
\limsup _{x \rightarrow x_{0}} \frac{\left|u(x)-u\left(x_{0}\right)\right|}{\left|x-x_{0}\right|} \leq 2 Q<\infty \quad \text { for a.e. } x_{0} \in \Omega \text {. }
$$


By Theorem 6.2, the weak solution $u$ is differentiable a.e. in $\Omega$.

\section{REFERENCES}

1. E. Acerbi and N. Fusco, Regularity for minimizers of non-quadratic functionals: The case $1<p<2$, J. Math. Anal. Appl.140 (1989), 115-135. MR 90f:49019

2. J. Björn, $L^{q}$-differentials for weighted Sobolev spaces, Michigan Math. J. 47 (2000), 151-161. MR 20001b:46849

3. J. Björn, Poincaré inequalities for powers and products of admissible weights, Ann. Acad. Sci. Fenn. Math. 26 (2001), 175-188.

4. B. Bojarski, Pointwise differentiability of weak solutions of elliptic divergence type equations, Bull. Acad. Polon. Sci. 33 (1985), 1-6. MR 87f:35063

5. E. De Giorgi, Un esempio di estremali discontinue per un problema variazionale di tipo ellittico, Boll. Un. Mat. Ital. 4 (1968), 135-137. MR 37:3411

6. H. Federer, Geometric Measure Theory, Springer-Verlag, Berlin-Heidelberg, 1969. MR 41:1976

7. J. Frehse, Una generalizzazione di un controesempio di De Giorgi nella teoria delle equazioni ellittiche, Boll. Un. Mat. Ital. 3 (1970), 998-1002. MR 43:2355

8. J. Frehse and U. Mosco, Variational inequalities with one-sided irregular obstacles, Manuscripta Math. 28 (1979), 219-233. MR 80i:49010

9. M. Fuchs, p-harmonic obstacle problems. I. Partial regularity theory, Ann. Mat. Pura Appl. 156 (1990), 127-158. MR 91m:49044

10. M. Fuchs, Smoothness for systems of degenerate variational inequalities with natural growth, Comment. Math. Univ. Carolin. 33 (1992), 33-41. MR 93e:49015

11. M. Giaquinta, Multiple Integrals in the Calculus of Variations and Nonlinear Elliptic Systems, Annals of Math. Studies 105, Princeton Univ. Press, Princeton, N. J., 1983. MR 86b:49003

12. M. Giaquinta and G. Modica, Almost-everywhere regularity results for solutions of non-linear elliptic systems, Manuscripta. Math. 28 (1979), 109-158. MR 80m:35033

13. E. Giusti, Regolarità parziale delle soluzioni di sistemi ellittici quasilineari di ordine arbitrario, Ann. Scuola Norm. Sup. Pisa (3) 23 (1969), 115-141. MR 52:6161

14. E. Giusti and M. Miranda, Sulla regolarità delle soluzioni deboli di una classe di sistemi ellittici quasi-lineari, Arch. Rat. Mech. Anal. 31 (1968), 173-184. MR 38:3574

15. P. Hajłasz and P. Koskela, Sobolev meets Poincaré C. R. Acad. Sci. Paris Sér. I Math. 320 (1995), 1211-1215. MR 96f:46062

16. P. Hajłasz and P. Koskela, Sobolev met Poincaré Mem. Amer. Math. Soc. 145 (2000). MR 2000j: 46063

17. P. Hajłasz and P. Strzelecki, On the differentiability of solutions of quasilinear elliptic equations, Colloq. Math. 64 (1993), 287-291. MR 94g:35082

18. J. Heinonen, T. Kilpeläinen and O. Martio, Nonlinear Potential Theory of Degenerate Elliptic Equations, Oxford University Press, Oxford, 1993. MR 94e:31003

19. S. Hildebrandt and K.-O. Widman, Variational inequalities for vector-valued functions, J. Reine Angew. Math. 309 (1979), 181-220. MR 81a:35023

20. P. A. Ivert, Regularitätsuntersuchungen von Lösungen elliptischer Systeme von quasilinearen Differentialgleichungen, Manuscripta Math. 30 (1979), 53-88. MR 82d:35045

21. J. Ježková, Boundedness and pointwise differentiability of weak solutions to quasi-linear elliptic differential equations and variational inequalitites, Comment. Math. Univ. Carolin. 35 (1994), 63-80. MR 95g:35071

22. O. John, J. Malý and J. Stará, Nowhere continuous solutions to elliptic systems, Comment. Math. Univ. Carolin. 30 (1989), 33-43. MR 90h:35050

23. G. Karch and T. Ricciardi, Note on Lorentz spaces and differentiability of weak solutions to elliptic equations, Bull. Polish Acad. Sci. Math. 45 (1997), 111-116. MR 97m:35047

24. O. A. Ladyzhenskaya and N. N. Ural'tseva, Linear and Quasilinear Elliptic Equations, 2nd ed., Nauka Press, Moscow 1973 (in Russian); Academic Press, New York, 1968 (English transl. of the 1st ed.). MR 58:23009; MR 39:5941

25. R. Landes, Some remarks on bounded and unbounded weak solutions of elliptic systems, Manuscripta Math. 64 (1989), 227-234. MR 88b:35158

26. H. Lewy and G. Stampacchia, On the regularity of the solution of a variational inequality, Comm. Pure Appl. Math. 22 (1969), 153-188. MR 40:816 
27. M. Meier, Boundedness and integrability properties of weak solutions of quasilinear elliptic systems, J. Reine Angew. Math. 333 (1982), 191-220. MR 83h:35042

28. J. H. Michael and W. P. Ziemer, Interior regularity for solutions to obstacle problems, Nonlinear Anal. 10 (1986), 1427-1448. MR 88k:35083

29. C. B. Morrey, Partial regularity results for non-linear elliptic systems, J. Math. Mech. 17 (1968), 649-670. MR 38:6224

30. O. Naselli Ricceri, The boundedness of solutions of variational inequalities of elliptic type with degeneration of type $A_{2}$, Boll. Un. Mat. Ital. C 4 (1985), 407-416. MR 87i:49020

31. J. Nečas and J. Stará, Principio di massimo per i sistemi ellittici quasilineari nondiagonali, Boll. Un. Mat. Ital. 6 (1972), 1-10. MR 47:3830

32. Yu. G. Reshetnyak, The differentiability almost everywhere of the solutions of elliptic equations, Sibirsk. Mat. Zh. 28 (1987), 193-195 (in Russian), Siberian Math. J. 28:4 (1987), 671-673 (English transl.). MR 90a:35037

33. J. Serrin, Local behavior of solutions of quasi-linear equations, Acta Math. 111 (1964), 247302. MR 30:337

34. E. M. Stein, Singular Integrals and Differentiability of Functions, Princeton University Press, Princeton, N. J., 1970. MR 44:7280

35. M. W. Stepanoff, Sur les conditions de l'existence de la différentielle totale, Mat. Sb., Rec. Math. Soc. Math. Moscou 32 (1925), 511-527.

36. P. Tolksdorf, Everywhere-regularity for some quasilinear systems with a lack of ellipticity, Ann. Math. Pura Appl. 84 (1983), 241-266. MR 85g:35053

37. K. Uhlenbeck, Regularity for a class of non-linear elliptic systems, Acta Math. 138 (1977), 219-240. MR 54:14031

38. N. N. Ural'tseva, On the regularity of solutions of variational inequalities, Uspekhi Mat. Nauk 42 no. 6(258) (1987), 151-174 (in Russian), Russian Math. Surweys 42:6 (1987), 191-219 (English transl.). MR 90c:35053

39. W. P. Ziemer, Weakly Differentiable Functions, Springer-Verlag, New York, 1989. MR 91e: 46046

Department of Mathematics, Lund Institute of Technology, P. O. Box 118, SE-221 00 Lund, SWEDEN

E-mail address: jabjo@maths.lth.se, jabjo@mai.liu.se 